\title{
Wissenschaftsministerin Schulze überreicht Verdienstkreuz am Bande an Prof. Otmar Schober
}

\author{
Auszeichnung für wissenschaftliches Engagement in der \\ Nuklearmedizin
}

Wissenschaftsministerin Svenja Schulze hat im Wege der Erstauszeichnung das Verdienstkreuz 1. Klasse des Verdienstordens der Bundesrepublik Deutschland an

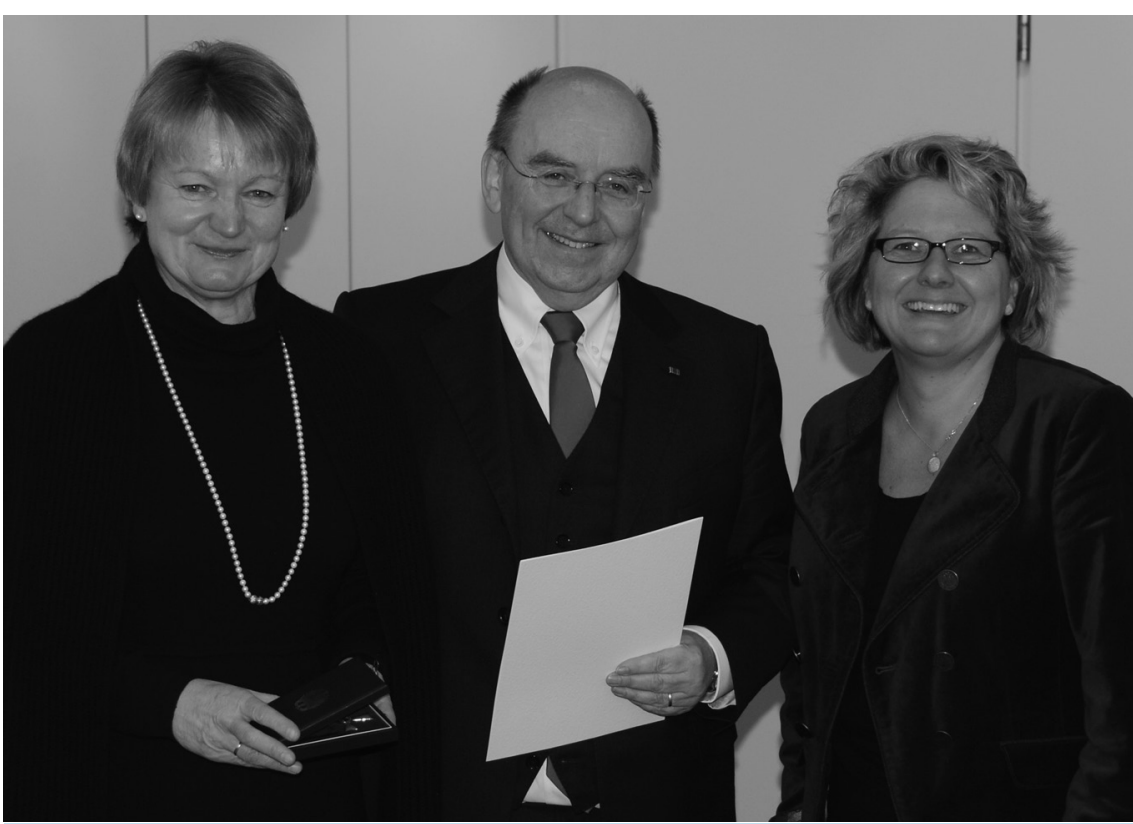

Prof. Dr. Dr. Otmar Schober mit seiner Frau, Dr. med. Anna Schober (links) und Wissenschaftsministern Dr. Svenja Schulze.

Prof. Dr. Dr. Otmar Schober verliehen. Die Ministerin betonte im Rahmen der Verleihung, dass Schober in seiner beruflichen Karriere stets weit über seine eigentlichen beruflichen Pflichten hinaus gewirkt habe. „Ihr Wirken war geprägt durch fachliche Kompetenz und das Mitfühlen anderen gegenüber“, so Ministerin Schulze.

In der Nuklearmedizin hat sich Schober durch die Mitgründung des European Institute of Molecular Imaging (EIMI) an der Universität Münster verdient gemacht. Das im Jahr 2007 gegründete Institut stellt die Schnittstelle zwischen Grundlagenforschung und Anwendung auf dem Gebiet der molekularen Bildgebung her. Es wird in einer neuen Konzeption interdisziplinär durch mehrere Fakultäten getragen. Otmar Schober hat im Laufe seiner wissenschaftlichen Karriere mehr als 300 Publikationen verfasst, bei denen die Ver- bindung von Grundlagenforschung und klinischer Anwendung im Mittelpunkt seines Interesses stand. Seine langjährige Arbeit in der Diagnostik des Schilddrü-

renamtlichen finanziell und persönlich unterstützt. Wissenschaftsministerin Schulze lobte das erfolgreiche und sichtbare Engagement Schobers im Ehrenamt wie in der Medizin. "Ihr Schaffen ist von gleichermaßen hoher wissenschaftlicher als auch gesellschaftspolitischer Bedeutung", bescheinigte die Ministerin Prof. Schober zur Auszeichnung mit dem Verdienstkreuz.

\section{Vita und Auszeichnungen $\nabla$}

Der 1948 in Strang (Bad Rothenfelde) geborene Otmar Schober ist seit 1988 Direktor der Klinik und Poliklinik für Nuklearmedizin an der Universität Münster. Von 1994 bis 1998 war er zudem Prorektor der Universität Münster. In den Jahren 1991 bis 1997 gehörte er dem Vorstand der Deutschen Röntgengesellschaft an und bildete einen wichtigen Brückenkopf zwischen Nuklearmedizin und Radiologie. Die Entwicklung des Deutschen Röntgenkongresses hat er vorangetrieben. 1995 bis 2003 war Schober Fachgutachter für die Nuklearmedizin bei der Deutschen Forschungsgemeinschaft. Der Deutschen Akademie der Technikwissenschaften gehört Prof. Schober seit 1996 als Mitglied des Themennetzwerkes Gesundheitstechnologie und Grundfragen der Technikwissenschaften an, zu dessen Sprecher er im Mai 2011 gewählt wurde. Ebenfalls seit 1996 ist Schober Mitglied der Nordrhein-Westfälischen Akademie der Wissenschaften und der Künste. Von 2002 bis 2010 war er zeitgleich Vorsitzender seiner Fakultät und der Ethikkommission der Ärztekammer Westfalen-Lippe. Im Jahr 2011 wurde er für die Verdienste um seine Fachgesellschaft zum Ehrenmitglied der Deutschen Gesellschaft für Nuklearmedizin ernannt. trauensdozent der Studienstiftung des Deutschen Volkes. Außerhalb seiner beruflichen Tätigkeit engagierte sich Prof. Schober in herausragender Weise gemeinsam mit seiner Frau für Schwerstkranke und Sterbende. Die beiden gründeten 2004 die “„chober-Stiftung“ für christliche Hospizarbeit. Auch die Palliativstation am Herz-Jesu-Krankenhaus in Münster-Hiltrup begleitet Prof. Schober seit ihren Anfängen. Hier hat die SchoberStiftung die Bildung eines Teams von Eh-
Mit freundlicher Genehmigung des Ministeriums für Innovation, Wissenschaft und Forschung des Landes Nordrhein-Westfalen (MIWF), Völklinger Straße 49, 40221 Düsseldorf, Deutschland 